\title{
Essential Roles of the Sperm Centrosome in Human Fertilization: Developing the Therapy for Fertilization Failure due to Sperm Centrosomal Dysfunction
}

\author{
Yukihiro Terada, ${ }^{1}$ Gerald Schatten, ${ }^{2}$ Hisataka Hasegawa ${ }^{1}$ and Nobuo Yaegashi ${ }^{1}$ \\ ${ }^{1}$ Department of Obstetrics and Gynecology, Tohoku University School of Medicine, Sendai, Japan \\ ${ }^{2}$ Department of Obstetrics, Gynecology and Reproductive Sciences, University of Pittsburgh School of Medicine, \\ Pittsburgh, PA, USA
}

\begin{abstract}
Fertilization is a lengthy process that culminates when the male and female pronuclei fuse in the oocyte cytoplasm. The final stage of fertilization is mediated by the sperm centrosome, which induces microtubule organization into the first mitotic spindle. Despite its critical role, only few functional analyses of the sperm centrosome have been performed until now. Here, we review the recent literature with regard to sperm centrosomal functions during human fertilization, as well as the development of functional assays for the human sperm centrosome. We then address various challenges for fertilization failure resulting from centrosomal dysfunction. Cytological analyses of oocytes that fail to complete fertilization following intracytoplasmic sperm injection (ICSI) have shown that some cases are associated with sperm centrosomal dysfunction. Human sperm can organize a sperm aster even within the oocytes of other mammals. This property has been utilized as a means of assessing the centrosome function. In some patients with teratozoospermia, the sperm does show evidence of impaired centrosomal function. Some clinical and basic challenges for overcoming the fertilization failure caused by sperm centrosomal dysfunction have been reported. The sperm centrosome plays an important role in the phase of the fertilization process after ICSI, i.e. within the oocyte's cytoplasm. The next generation of assisted reproductive technologies (ART) will likely incorporate analyses of sperm centrosomal function as well as techniques designed to counter sperm centrosome dysfunction.
\end{abstract}

Keywords: assisted reproductive technique; fertilization; intracytoplasmic sperm injection; infertility; sperm centrosome

Tohoku J. Exp. Med., 2010, 220 (4), 247-258. (C) 2010 Tohoku University Medical Press

Assisted reproductive technology (ART) has achieved worldwide acceptance over the past 30 years (Sutcliffe and Ludwig 2007). In some industrialized nations, one in 50 children is born following ART (Manipalviratn et al. 2009). Thus, continued evaluation and improvement of ART is critical to ensure that it remains safe and effective. In the 1980s, use of ART was widespread. The introduction of intracytoplasmic sperm injection (ICSI) (Palermo et al. 1992) enabled couples with severe male factor infertility such as oligozoospermia to achieve pregnancies. Additionally ICSI promised to improve the ART efficiency for couples with milder forms of male factor infertility. However, by the end of 2006, the pregnancy rate following ART remained at $20 \%$, and the live birth rate was only $15 \%$ resulting in concerns regarding patient satisfaction (Manipalviratn et al. 2009).
Fertilization is the series of events uniting the male and female genomes into a single entity followed by the formation of the first mitotic spindle. ICSI, while a significant technological advance, only assists with passage of the sperm through the cumulus cells, through the zona and into the oocyte cytoplasm. ICSI does not, however, address defects either in the steps leading up to genome fusion or in the process of fertilization itself. These events have been termed post-ICSI events in fertilization (post-ICSI). While post-ICSI events are clearly significant to achieving a viable embryo, the mechanisms involved in post-ICSI are not well studied. Furthermore, it is unclear whether disorders of post-ICSI are involved in human sterility.

In this article, we review the current literature pertaining to human sperm centrosomal function and how centrosomal dysfunction relates to human sterility. We then

Received February 17, 2010; revision accepted for publication March 3, 2010. doi:10.1620/tjem.220.247

Correspondence: Yukihiro Terada M.D., Ph.D., Department of Obstetrics and Gynecology, Tohoku University School of Medicine, 1-1, Seiryo-machi, Aoba-ku, Sendai 980-8574, Japan.

e-mail: terada@mail.tains.tohoku.ac.jp

Dr. Yukihiro Terada is a recipient of the 2009 Gold Prize, Tohoku University School of Medicine. 
address the challenges encountered in developing treatments for fertilization failure due to the sperm centrosomal dysfunction along with improved diagnostics.

\section{Post-ICSI events in fertilization: role of the sperm centrosome in maternal and paternal genome fusion}

It is unclear whether in vitro fertilization (IVF) is effective for infertility related to all fertilization disorders, as fertilization disorders in IVF have not been precisely defined. However, the occurrence of fertilization, itself, can be confirmed following IVF. Several interventions for IVF fertilization failure, including sperm manipulation, have been investigated (Yanagida et al. 1999; Murase et al. 2004; Tomashov-Matar et al. 2005; Nasr-Esfahani et al. 2008; Terada et al. 2009a). However, most did not develop sufficiently well so as to gain acceptance into standard ART protocols.

Before treatments for fertilization disorders may be developed, the basic molecular mechanisms of fertilization must be understood. Several molecular mediators associated with sperm-oocyte fusion have been identified in laboratory animals (Ikawa et al. 2008). Of these, only IZUMO appears essential as knockout of this gene in the mouse results in complete fertilization failure (Inoue et al. 2005). We examined the expression of IZUMO in the sperm of infertile patients. In these patients, routine IVF was unsuccessful; however, fertilization was achieved with ICSI. IZUMO expression was measured in all cases and surprisingly, all expressed it. This underscores the complexity of the fertilization process and illustrates that fertilization failure is unlikely to be the result of a defect in a single factor (Hayasaka et al. 2007).

Since its introduction in 1992, ICSI has become a standard practice worldwide. It is effective in circumventing disorders of sperm-oocyte fusion, thereby enabling couples in which traditional IVF had failed to become pregnant (Palermo et al. 1992). While ICSI has overcome some defects in fertilization, a number of patients still fail to achieve fertilization even with ICSI (Fig. 1). It is likely that the events downstream of sperm injection, the aforementioned post-ICSI, are responsible. Little is understood regarding post-ICSI events (Schatten et al. 1998; Terada et al. 2003; Terada 2007). Cellular analysis in human zygotes clinically diagnosed as 'unfertilized' suggests that several of these zygotes actually arrested at a point in the "post-ICSI events in fertilization" (Rawe et al. 2000; Kovacic and Vlaisavljevic 2000).

The human sperm must perform three vital functions after entering the oocyte. It contributes the male genome, awakens the quiescent oocyte, and crystallizes the motility apparatus that unites the sperm and oocyte nuclei (Schatten 1994; Simerly et al. 1995; van Blerkom et al. 1995). The sperm carries both mitochondria and microRNA into the oocyte; however, the role of these in the zygote is unknown (Yan et al. 2008). It is clear that the sperm serves a vital role beyond being a carrier of genetic information. It is defects in these critical downstream functions which cannot be compensated for with ICSI.

The events following entry of the sperm into the oocyte have been addressed in several studies. In an excellent kinetic study using time-lapse cinematography, Mio and colleagues have described the various phenomena occurring within the fertilized human oocyte (Mio and Maeda 2008). One longstanding question regarding the process is whether a fertilization cone is formed (Tsuiki et al. 1986). Indeed, it has now been shown that following fertilization, dynamic movement of male and female pronuclei towards the center of egg has been shown (Tsuiki et al. 1986).

The microtubule-based system is a cytoskeletal structure that is critical to the movement of the male and female genomes (Schatten 1994). Microtubules are polar structures, one end of which is embedded in and stabilized by the microtubule-organizing center (MTOC). In the majority of animal cells, the MTOC is the centrosome, which consists of a pair of centrioles surrounded by fibrous and/or amorphous pericentriolar material (PCM). The centrosome is important in stabilizing cell movement, establishing cell polarity, and regulating the cell cycle (Voribjev and Chenstov 1982). The microtubule nucleating activity of MTOCs requires the presence of $\gamma$-tubulin, which is mainly associated with PCM (Simerly et al. 1999).

In most species, with the exception of rodents, the centrosome is provided by the sperm, and it forms a monopolar array of microtubules known as the sperm aster (Sathananthan et al. 1997). The sperm aster is essential for pronuclear movement for the union of the male and female genomes. Supernumerary centrosomes account for the lethality of polyspermy (Asch et al. 1995).

Microtubule organization in human fertilization has been documented by Simerly and colleagues (Simerly et al. 1999). In human fertilization, shortly after sperm entry, astral microtubules assemble around a sperm head to form the "sperm aster", as the inseminated oocytes complete the second meiotic division and extrude the second polar body. As the male pronucleus continues to decondense in the cytoplasm, the microtubule sperm aster enlarges, enveloping the male pronucleus. This organization of microtubules from the sperm centrosome is essential for the movement and fusion of the male and female pronuclei. Following syngamy, the sperm centrosome replicates and splits, and organizes the bipolar array that forms the mitotic spindle required for cleavage. These sperm centrosomal functions are also critical in the "motility" of the post ICSI events in fertilization (Palermo et al. 1997).

In rodents, the paternal centrosome degenerates during spermiogenesis (Manandhar et al. 2005), and microtubules assemble in association with the maternal pericentriolar material. The maternal centrosomes remain dispersed as the cytoplasmic MTOCs due to the absence of centrioles, and form multiple asters during the pronuclear stage. These 
cytoplasmic asters are attracted to the surfaces of both the male and female pronuclei and are involved in pronuclear movement (Schatten et al. 1985). This illustrates that the pattern of centrosome inheritance during fertilization is species specific.

Abnormal microtubule organization in human zygotes, which are clinically diagnosed as "unfertilized," suggests that centrosomal function contributes to fertilization failures after proper sperm entry (Rawe et al. 2000; Kovacic and Vlaisavljevic 2000). Additionally, the function of the zygotic centrosome varies among bulls during IVF and this variation affects male fertility (Navara et al. 1996). These reports suggest that sperm centrosomal function might affect fertility in human reproduction and that an appropriate assay to examine centrosomal inheritance and function would benefit ART. Therefore, proper function of the human sperm centrosome is thought to be essential for human fertility. The direct assessment of human centrosome function, however, has proven to be challenging (Terada et al. 2003).

Schatten et al. have shown that calcium oscillations during oocyte activation trigger the release of the tail microtubule from the basal body by centrin-induced cleavage. Subsequently, the sperm centrosome is then phosphorylated, while the disulfide bonds are simultaneously reduced (Schatten 1994). The sperm centrosome then binds additional $\gamma$-tubulin derived from the oocyte cytoplasm, forming a centriole. The resulting halo of $\gamma$-tubulin nucleates the microtubules, which assemble into the sperm aster. The microtubule nucleating activity of MTOCs requires the presence of $\gamma$-tubulin. While both parents contribute $\gamma$ tubulin, inheritance is preferentially maternal. Recruitment of maternal $\gamma$-tubulin to the sperm centrosome occurs after sperm incorporation in vivo. It may also be triggered in vitro following exposure to a Xenopus laevis cell-free extract, particularly after sperm "priming" induced by disulfide bond reduction (Simerly et al. 1999). Other proteins may also be involved in microtubule nucleation. Centrin, a ubiquitous calcium-sensitive, biparentally contributed, centrosomal component, severs axonemal microtubules from their associated basal bodies, potentially functioning in centrosome duplication. Centrin abnormalities may lead to fertilization failure or incomplete embryonic development (Salisbury 1995; Schiebel and Bornens 1995; Levy et al. 1996). Pronuclear migration and apposition require cytoplasmic dynein, a microtubule-based motor protein, and the cofactor dynactin. Together, these establish the directionality of female pronuclear movement and the dynamics of its motion along the sperm aster microtubules (Payne et al. 2003).

\section{Fertilization failures after ICSI}

Cytological analyses of oocytes are one way to assess which processes are arrested in the case of fertilization failure following ICSI. For example, the status of the second polar body provides some insight into why fertilization has not occurred. Assessment of the polar body, however, is not currently used clinically. In most ART protocols oocytes are usually cultured for several days following ICSI. When oocytes are finally deemed unfertilized, many no longer have the structural integrity to withstand the fixing process. While some centers do describe incorporating a cytological analysis into ART protocols (Rawe et al. 2000; Kovacic and Vlaisavljevic 2000; Ramalho-Santos et al. 2004), acceptance has not been widespread (Terada 2007).

Kovacic and Vlaisavljevic (2000) and Rawe et al. (2000) have shown that half of the oocytes that fail to fertilize following ICSI demonstrate disorders of activation. Another cause of arrest that may be considered is failure of the injection itself. Approximately $20 \%$ of activated oocytes show abnormalities in other processes beyond activation, including microtubule dynamics (Rawe et al. 2000, $2002 b$ ). Among them, about $20 \%$ of oocytes show pronuclear formation but lack subsequent pronuclear movement, and fail to form the sperm aster. These cases are assumed to have a functional problem with the sperm centrosome. The analysis of oocytes arrested following fertilization is challenging; however, as illustrated by Rawe et al. cytological analyses may provide valuable insight into the causes of fertilization failure (Rawe et al. 2002a; Rawe and Chemes 2009).

We have reported a case that required eight cycles of ICSI prior to achieving successful fertilization. Those oocytes, which were not fertilized following ICSI, were fixed and analyzed with respect to their nuclear phase and microtubule organization (Fig. 1). Among the twelve oocytes examined, two (16.7\%) showed pronuclear formation; however, the sperm aster was not observed (Terada et al. 2009a). While not a universal finding, it is possible to identify those oocytes that fail to fertilize secondary to sperm aster dysplasia and sperm centrosomal dysfunction.

\section{Development of a functional assay for the human sperm centrosome}

Recently, a number of novel methods have been reported to examine sperm centrosomal function using a heterologous ICSI system in which human sperm is microinjected into either a rabbit or bovine oocyte (Nakamura et al. 2001; Terada et al. 2002, 2004a). After human sperm incorporation into a rabbit or bovine oocyte, we have observed that the sperm aster is organized from the sperm centrosome, and the sperm aster is enlarged as the sperm nucleus undergoes pronuclear formation (Fig. 2). The human sperm aster formation rate at $6 \mathrm{~h}$ post-ICSI is $36.1 \%$ in rabbit oocyte and $60.0 \%$ in bovine oocyte (Terada et al. 2000; Terada et al. 2004b). The microtubule organization in these systems is derived from the paternal centrosome during fertilization, and mimics that seen in normal human fertilization (Simerly et al. 1995; Yellera-Fernandez et al. 1992; Navara et al. 1994). In 2004, we reported human sperm aster formation rates in rabbit oocytes after heterologous microinjection, using sperm from infertile men. In 

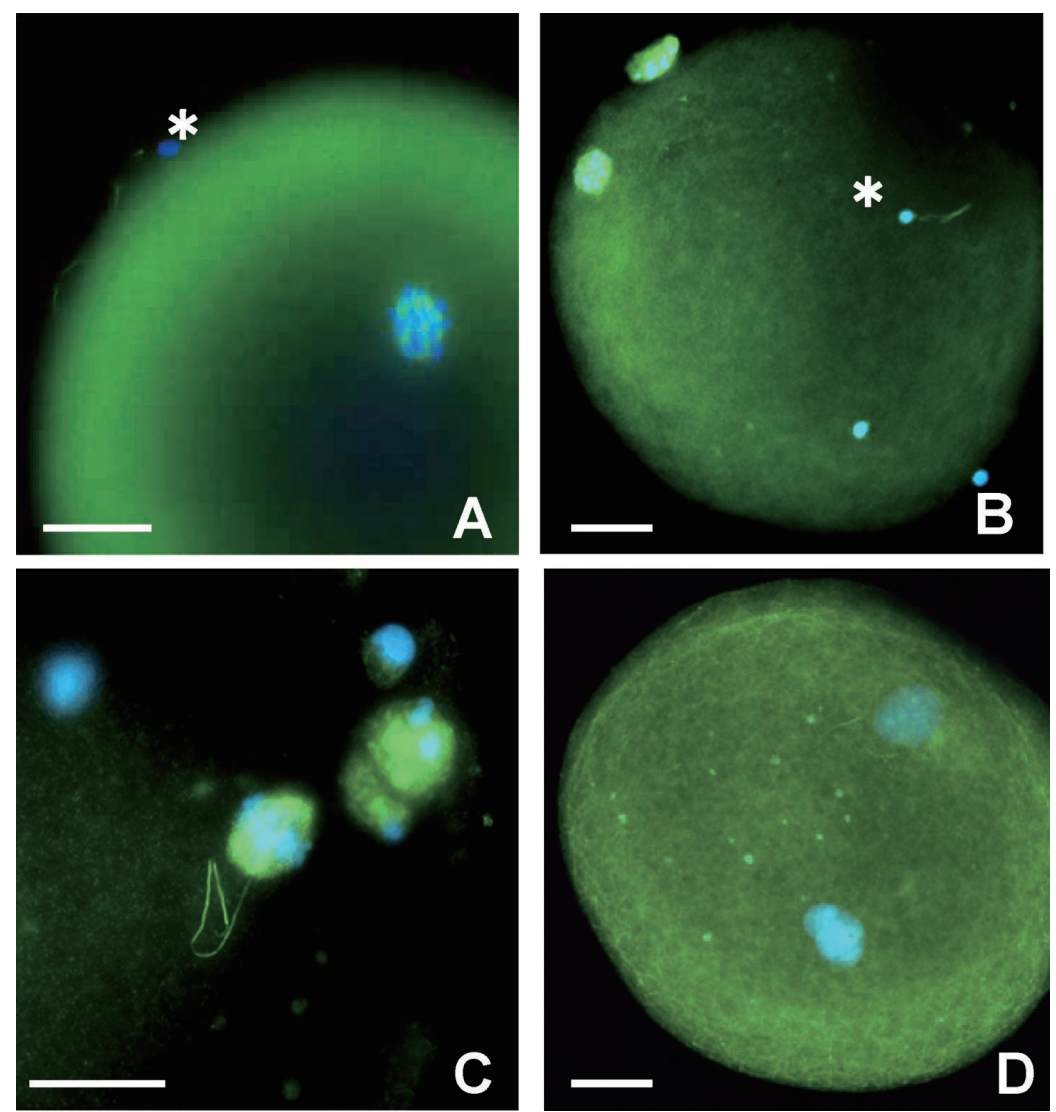

Fig. 1. Fertilization failure not overcome by ICSI.

Human oocytes, judged to be unfertilized 30-48h after ICSI, were fixed and stained in order to visualize the DNA and microtubules with immunofluorescent microscopy.

A: Technical ICSI failure: The sperm nucleus is present outside of the cytoplasmic membrane (asterisk), probably due to a failed injection. The oocyte contains an intact M2 meiotic spindle. B: Activation failure (1): The sperm nucleus is slightly decondensed (asterisk) and the oocyte contains an intact M2 meiotic spindle within its cytoplasm. C: Activation failure (2) Premature condensation of chromosomes (PCC): The sperm centrosome organizes a spindle only around the male genome. The oocyte's chromosomal arrangement is unaffected. D: Oocytes arrested by sperm centrosomal dysfunction: The oocyte has been activated, forming two pronuclei; however, the pronuclear centration was arrested without the formation of the radial array of microtubules from the sperm centrosome (sperm aster). This is a characteristic feature of the fertilization arrest that is caused by sperm centrosomal dysfunction. (DNA: blue; microtubules: green) [Reproduced with modifications from Terada et al. (2009a): Fertil. Steril. 91, 1271-1272, 2009.]
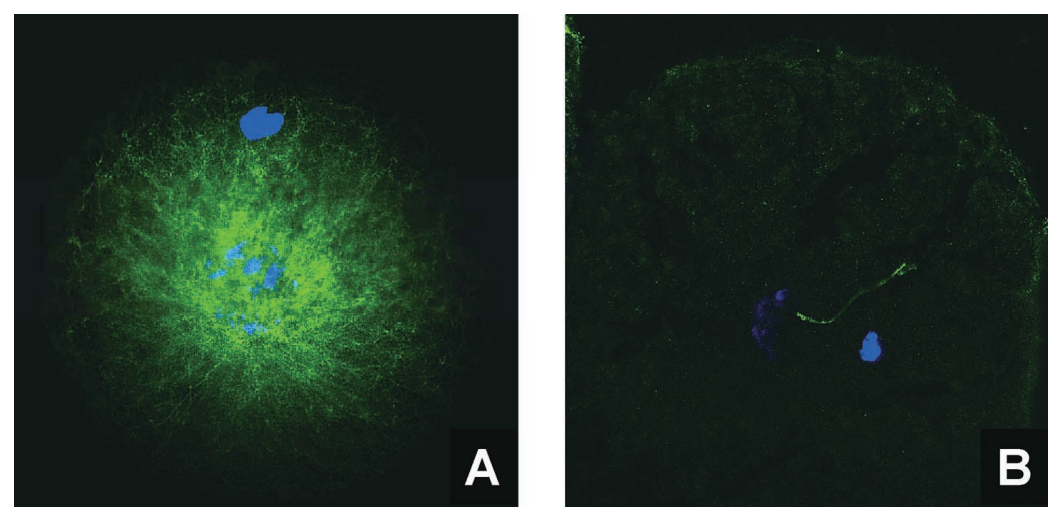

Fig 2. Formation of human sperm aster in the bovine oocyte cytoplasm, an assay of human sperm centrosomal function.

A: Human sperm was microinjected into matured bovine oocyte with a Piezo-micromanipulator. $6 \mathrm{~h}$ after microinjection, oocytes were fixed and the DNA configuration and microtubule organization assessed. 60-70\% of fertile human sperm will organize a radial array of microtubules from the sperm centrosome (sperm aster). B: Absence of human sperm asters $6 \mathrm{~h}$ after microinjection. (DNA: blue; microtubules: green)

[Original data, Terada, Y., Hasegawa, H., and Yaegashi, N.] 
rabbit oocytes, human sperm aster formation rates correlate with cleavage rates, but do not reflect the rate of pronuclear formation in clinical IVF (Terada et al. 2004a). We have also assessed the centrosomal function of sperm from infertile patients by heterologous injection into bovine oocytes. Human sperm aster formation rates assessed by bovine oocytes are independent from clinical semen characteristics and pronuclear formation rates on clinical IVF. However, significant differences in sperm aster formation rates are correlated with the embryonic cleavage rates and pregnancy after clinical ART (Yoshimoto-Kakoi et al. 2008). These results indicate that centrosomal function contributes to the fertilization process downstream from pronuclear formation. Furthermore, these studies touch on the importance of human sperm centrosomal dysfunction in infertility.

Globozoospermia is one type of teratospermia. Globozoospermia is defined by the presence of sperm with round heads that lack the acrosome and acrosomal enzymes and have a disorganized mid-piece (Singh 1992). The roundheaded sperm cannot penetrate the oocyte zona pellucida and is therefore incapable of fertilization (Rybouchkin et al. 1996, 1997). We assessed sperm centrosomal function in round-headed sperm following heterologous ICSI, using bovine oocytes. The rate of sperm aster formation in the oocytes injected with round-headed sperm was $15.8 \%$ and was significantly lower than the rate of aster formation in the oocytes injected with fertile donor sperm (Table 1). Ethanol activation improved the rate of male pronuclear formation to $84.9 \%$ in oocytes injected with the roundheaded sperm. However, a corresponding increase in sperm aster formation $(32.3 \%)$ was not seen following ethanol activation (Nakamura et al. 2002). These results suggest that sperm centrosomal function is independent of a sperm's ability to activate the oocyte (Dam et al. 2007). Dysplasia of the fibrous sheath (DFS), a rare form of teratospermia, results in infertility. DFS sperm, which are immotile due to deformities from the mid-piece to tail (Chemes et al. 1987), also exhibit sperm centrosomal dysfunction. Both of these abnormalities may be causes of infertility. These defects often cannot be overcome with ICSI. The human sperm aster formation rate in bovine oocytes injected with DFS sperm is less than $20 \%$ (Rawe et al. 2002b, Table 1).

The above observations suggest that the sperm from men with congenital teratospermia have centrosomal dysfunction (Fig. 3, Table 1). The cause of centrosomal dysfunction in this setting is uncertain; however, the morphological anomalies in the mid-piece of the sperm may play a role (Chemes and Rawe 2002a). Using immunofluorescent

Table 1. Rate of sperm aster organization in bovine egg injected with sperm from a globozoospermia donor or Dysplasia of the fibrous sheath (DFS) donor or fertile donor.

\begin{tabular}{lcc}
\hline & Egg & $\begin{array}{c}\text { Sperm aster } \\
\text { organization rate }\end{array}$ \\
\hline Globozoospermia & 19 & $3(15.8 \%)^{\mathrm{a}}$ \\
DFS & 25 & $4(16.0 \%)^{\mathrm{a}}$ \\
Fertil donor & 22 & $15(68.2 \%)^{\mathrm{b}}$ \\
\hline
\end{tabular}

a vs $\mathrm{b} P<0.001$

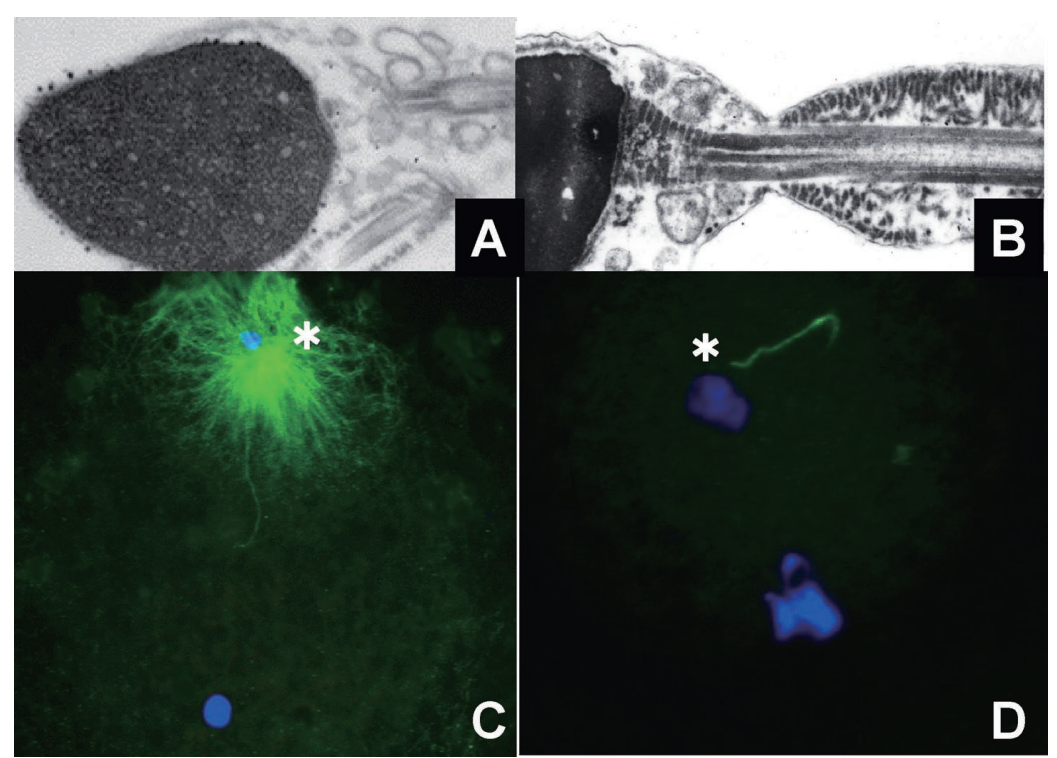

Fig. 3. Impaired sperm centrosomal function in men with teratozoospermia.

Scanning electron micrograph images of human sperm from teratozoospermic patients (A,B). A: Globozoospermia; B: Dysplasia of the Fibrous Sheath. Disorganization of sperm mid-piece is evident in both disorders. C: Human sperm aster organized in the cytoplasm of a bovine oocyte following injection with sperm from a globozoospermia patient (asterisk). D: Sperm from a DFS patient fails to form a sperm aster (asterisk) following injection into a bovine oocyte (DNA: blue; microtubules: green)

[Reproduced with modifications from Nakamura et al. (2002): Hum. Reprod.17, 2930-2934, 2002 and from Rawe et al. (2002b): Hum. Reprod. 17, 2344-2349, 2002.] 
staining, we have described the expression of centrin (Salisbury 1995), a functional centrosomal protein in normal and DFS sperm. Centrin is expressed in $100 \%$ of normal sperm in the mid-piece. In contrast, only $2 \%$ of DFS sperm express it at the mid-piece (Nakamura et al. 2005). These observations imply that defects in centrosome function may be the result of structural and functional problems within the sperm.

Currently, protocols for ART do not include an assessment of sperm centrosomal dysfunction. It is becoming clear that this is one area in which interventions may be targeted in order to overcome at least some cases of post-ICSI fertilization failure.

\section{How to treat infertility resulting from sperm centrosomal dysfunction}

As previously discussed, sperm centrosome dysfunction has been identified as the cause of infertility in some cases with IVF fertilization failure (Terada et al. 2003). The confirmation of a sperm aster within the oocyte is technically challenging. Therefore, the prevalence of sperm centrosomal dysfunction has not been established in patients undergoing IVF for infertility. Regardless, treating centrosome dysfunction will likely result in an increase in the efficiency of ART (Terada et al. 2003; Terada 2007). The following two challenges can be candidates for treatment against sperm centrosomal dysfunction. The first candidate is to restore the intrinsic sperm centrosomal function via chemical treatment. The other employs the transplantation of an isolated normal sperm centrosome (Mitchison and Kirschner 1984; Picard et al. 1987).

Nakamura et al. (2005) describe the chemical treatment of DFS sperm as an approach to restore centrosomal function. Human sperm have disulfide bonds within the head and pericentriolar regions (Seligman et al. 1994; Simerly et al. 1999; Tateno and Kamiguchi 1999). Dithiothreitol (DTT), which induces the reduction of disulfide bonds, is effective for unraveling the sperm centrosome. Paclitaxel $\left(\right.$ Taxol $\left.^{\mathrm{TM}}\right)$, which acts as a cytoskeleton stabilizer, is frequently used for studying cytoskeletal dynamics (Hewitson et al. 1997; Mailhes et al. 1999). Paclitaxel enhances microtubule polymerization within the oocyte cytoplasm. We attempted to restore the sperm centrosome function of aster formation, which extremely occur only at a low rate in patients with DFS. The combination of DTT and paclitaxel, while sufficient to induce microtubule organization in dead sperm from a fertile donor following heterologous ICSI, was not effective for DFS sperm.

These results indicate that sperm centrosomal function may be induced by the treatment of sperm with DTT before ICSI and of oocytes with paclitaxel after ICSI. DFS sperm, however, likely exhibit too severe a dysfunction of the sperm centrosome corrected by this chemical manipulation (Nakamura et al. 2005).

\section{Microtubule organization during oocyte cleavage without a sperm centrosome}

Parthenogenesis is an extraordinary process in which the activated oocyte initiates full development, resulting in a sexually mature adult without a paternal contribution. Examination of microtubule organization during parthenogenesis illustrates how the oocyte develops a functional MTOC. In some non-rodent mammals, such as cows (Navara et al. 1994; Shin and Kim 2003), rabbits (PintoCorreia et al. 1993), pigs (Kim et al. 1996), and marsupials (Breed et al. 1994), parthenotes exhibit disarrayed microtubules in the cytoplasm shortly after artificial activation. This suggests that these mammalian oocytes are able to form a functional centrosome without contributions from the sperm. However, a role for the maternal centrosome in microtubule organization and pronuclear movement has not been fully elucidated. Comparing the mitotic events that occur when the sperm centrosome is present with division when it is absent, elucidates the functional relationship between the sperm centrosome and the oocyte MTOC. Understanding this functional relationship will facilitate the treatment of centrosome dysfunction.

We have previously detailed the dynamics of microtubules and the distribution of $\gamma$-tubulin, a major component of the maternal centrosome, during the first interphase of bovine parthenogenesis (Morito et al. 2005). In chemically activated bovine parthenotes treated with paclitaxel, cytoplasmic microtubule asters become organized and microtubules dynamically radiate towards the female pronucleus. In bovine oocytes, microtubule patterns correlate well with pronuclear movement to the cell center. Microtubules aggregate at regions of high $\gamma$-tubulin concentration, which are not present until the first interphase of bovine parthenogenesis. These findings indicate that $\gamma$-tubulin is responsible for microtubule organization as the maternal centrosome in bovine parthenogenesis. Our results support the theory that the maternal centrosome has the ability to fulfill a role as a functional centrosome, despite its impairment in the nucleation of microtubules and a lack of ability to self-replicate or form spindle poles without sperm centrosomal contributions (Fig. 4).

Centrosomal inheritance during rabbit fertilization is thought to be biparental (Terada et al. 2000). However, the specific roles of the paternal and maternal centrosome have not been elucidated until recently (Longo 1976; PintoCorreia et al. 1994; Wu and Palazzo 1999). To define the role of the sperm centrosome in rabbit fertilization, paternal centrosome dysfunction model was examined. The sperm centrosome was first removed by sonication from the sperm nucleus. ICSI was then performed either with an isolated sperm head or with an intact sperm, and microtubule organization examined. No sperm aster formation was seen in the oocytes following injection with an isolated sperm head, and microtubule organization without a distinct nucleation site between the male and female pronuclei was observed. 
A

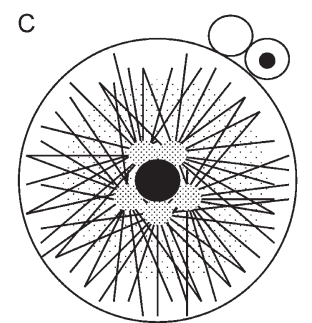

B
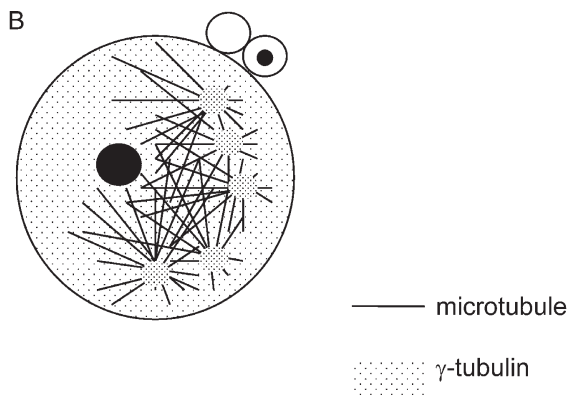

D

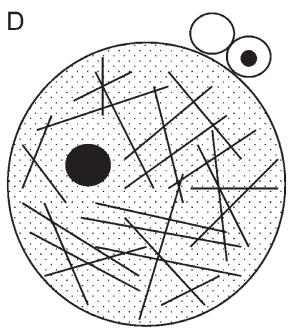

maternal centrosome ( $\gamma$-tubulin)

Fig. 4. A schematic diagram of microtubule dynamics and female pronuclear positioning during the first interphase of bovine parthenogenesis.

Several microtubule asters have formed in the cortical region of the cytoplasm in the early pronuclear period (Fig. 4A). At this stage, microtubules radiate from each focus toward the female pronucleus, connecting through a microtubule network (Fig. 4B). At the late pronuclear stage, microtubules extend from each focus to fill the cytoplasm, aggregating around the female pronucleus (Fig. 4C). The formation of a mesh-like network of disarrayed microtubules is similar to that seen in untreated oocytes (Fig. 4D). Microtubule asters are only observed in half of the parthenotes $2 \mathrm{~h}$ after activation. [Reproduced with midifications from Morito et al. (2005): Biol. Reprod. 73, 935-942, 2005.]

In contrast, sperm aster formation was observed in oocytes following ICSI with an intact sperm. The first mitotic spindle was organized after ICSI with the isolated sperm head, as observed in oocytes with an intact sperm (Morita et al. 2005). These findings indicate that the MTOC exists in the oocyte cytoplasm. The oocyte MTOC is sufficient to complete the microtubule organization that is essential for genomic union and formation of the first mitotic spindle. In addition, since aster formation was seen following ICSI with an intact sperm, the maternal centrosome seemed to fulfill the paternal sperm centrosome's function when the sperm centrosome was absent. These studies have shown that a functional centrosome or MTOC is present in the oocytes of some mammals; however, when a sperm aster is formed by normal fertilization, a corresponding cytaster derived from the oocyte MTOC is not observed. Regardless, understanding the role of the oocyte MTOC will likely also be useful in the development of interventions targeting sperm centrosomal dysfunction.

While much has been learned from the study of animal models of fertilization, we have also studied microtubule organization during human parthenogenesis. Human oocytes were obtained from therapeutic laparoscopic ovarian drilling in infertile, polycystic ovarian syndrome patients and matured in vitro (Cavilla et al. 2008). Mature human oocytes were activated with $5 \mu \mathrm{M}$ ionomycin for $5 \mathrm{~min}$ and then incubated with $2 \mathrm{mM}$ 6-dimethylamino purine (6-DMAP) (Paffoni et al. 2007). Two hours post activation, multiple microtubule asters (cytasters) were observed in the oocyte cytoplasm. Six hours after activation, microtubules without a distinct nucleation site were organized around a pronucleus that was moving to the oocyte center. Twelve hours after activation, the first mitotic spindle was organized in the human parthenote (Fig. 5). These observations indicate that multiple MTOC, which function in a manner similar to a sperm centrosome, are present in the human oocyte cytoplasm during parthenogenesis (Terada et al. 2009b).

\section{Functional relationships of the sperm centrosome}

Injection of a normal sperm centrosome at the time of ICSI may compensate for infertility caused by centrosome dysfunction. Indeed, this novel strategy has been explored and been shown to be effective both in terms of centrosome isolation (Mitchison and Kirschner 1984) and transplantation (Picard et al. 1987). One important factor to consider in transplanting centrosomes is the interaction between the defective and the normal transplanted centrosome. As discussed previously, the oocyte cytoplasm functions as a MTOC when the sperm centrosome is absent (Morito et al. 2005; Terada et al. 2009b). This process is suppressed when the sperm centrosome is functioning normally (Simerly et al. 1995; Nakamura et al. 2001). Generally, cells will not tolerate multiple active centrosomes because they induce multipolar spindles (Brinkley and Goepfert 1998). Fertilization in Spisula solidissimia oocytes results in cells with three active centrosomes, two maternal and one paternal, but the function of the paternal centrosome is selectively shut off, possibly because the embryos can identify and 


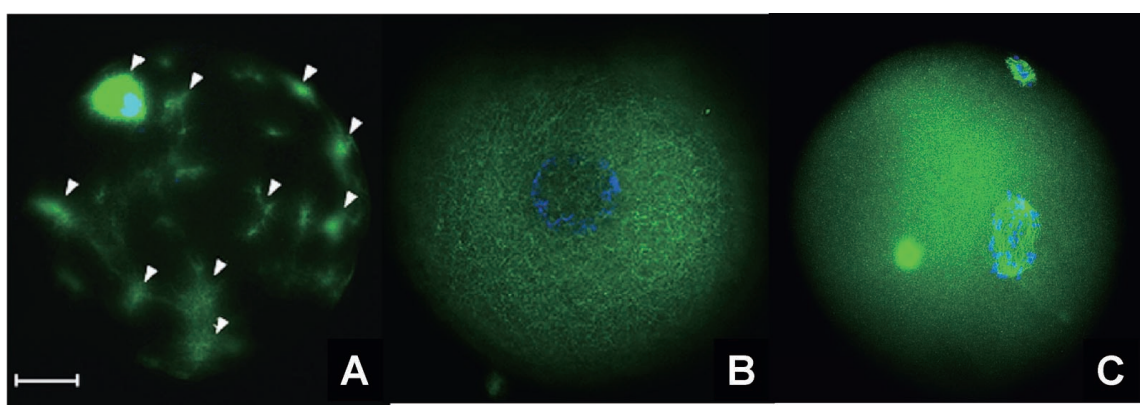

Fig. 5. Microtubule organization during human parthenogenesis.

Mature human oocytes were activated with $5 \mu \mathrm{M}$ ionomycin for $5 \mathrm{~min}$ and then incubated with $2 \mathrm{mM}$ 6-dimethylamino purine. Microtubule organization and DNA configuration of these oocytes were then examined. A: Two hours post activation: multiple asters of microtubules (cytasters) are observed. B: Six hours after activation, microtubules without a distinct nucleation site are organized around a pronucleus that is moving to the oocyte center. C: Twelve hours after activation: The first mitotic spindle is organized in the human parthenote. (DNA: blue; microtubules: green)

[Reproduced with midifications from Terada et al. (2009b): Fertil. Steril. 91, 1271-1272, 2009.]

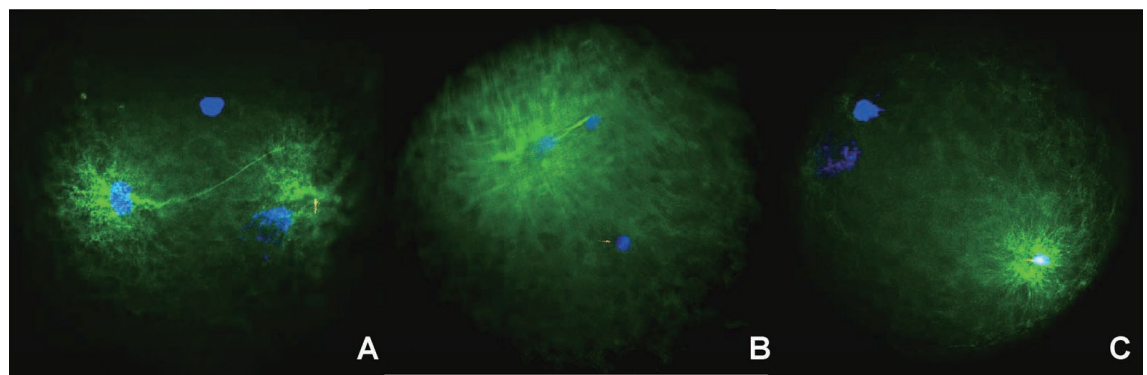

Fig. 6. Human sperm aster formation after injection of multiple human sperm into a bovine oocyte.

Two human sperm were microinjected 30 minutes apart into an oocyte. The second sperm was labeled with MitoTracker orange prior to injection. A: Two sperm aster are organized in bovine oocyte cytoplasm. B: A sperm aster organized only from the centrosome of the first sperm injected. C: A sperm aster organized only from the centrosome of the second sperm injected.

(DNA: blue; microtubules: green; sperm mitochondria: red)

[Original data, Terada, Y., Hasegawa, H., and Yaegashi, N.]

control the function of the centrosomes (Wu and Palazzo 1999). These reports suggest that a functional relationship between centrosomes exists. If a normally functioning centrosome is simultaneously present with an abnormal centrosome, the potential for interaction exists and must be considered (paternal vs. maternal, paternal vs. paternal).

In nature, fertilization occasionally does occur with multiple sperm penetrating the oocyte cytoplasm resulting in polyspermy. However, while polyspermy has been studied, until recently it has been unclear what interactions occur, when an oocyte is fertilized with multiple sperm (Asch et al. 1995; Iwao et al. 1997; Suzuki et al. 2003). We examined the sperm aster formation after injecting multiple human sperm into a bovine oocyte. If paternal centrosomes function independently of one another, transplantation of a normal centrosome is a viable option for treating centrosomal dysfunction. We examined sperm aster formation after heterologous ICSI with multiple human sperm into a bovine oocyte (Terada et al. 2009c). When two fertile human sperm were simultaneously microinjected into different regions of the same bovine oocyte cytoplasm, no difference in sperm aster formation rate was observed com- pared to cases in which a single sperm was injected. Two human sperm were also microinjected into bovine oocyte 30,60 or 120 min apart from one another, and again no difference in sperm aster formation rates was observed. Among oocytes in which one sperm aster was organized, there was no bias towards the sperm that was injected first or second. These findings indicate that when multiple human sperm are present in a single oocyte cytoplasm, each centrosome can function without being influenced by the other. Therefore, centrosome transplantation is one potential treatment for centrosomal dysfunction (Fig. 6).

\section{Strategy for the selection of the sperm with normal centrosomal function}

In this report we have addressed infertility related to sperm centrosome dysfunction and have explored possible treatment strategies. However, all currently proposed interventions depend on complicated gamete manipulations (Nakamura et al. 2005; Terada et al. 2009a), the safety and efficacy of which are not known. Further studies are needed to refine and standardize these techniques prior to their incorporation into standard ART protocols. We have previously 

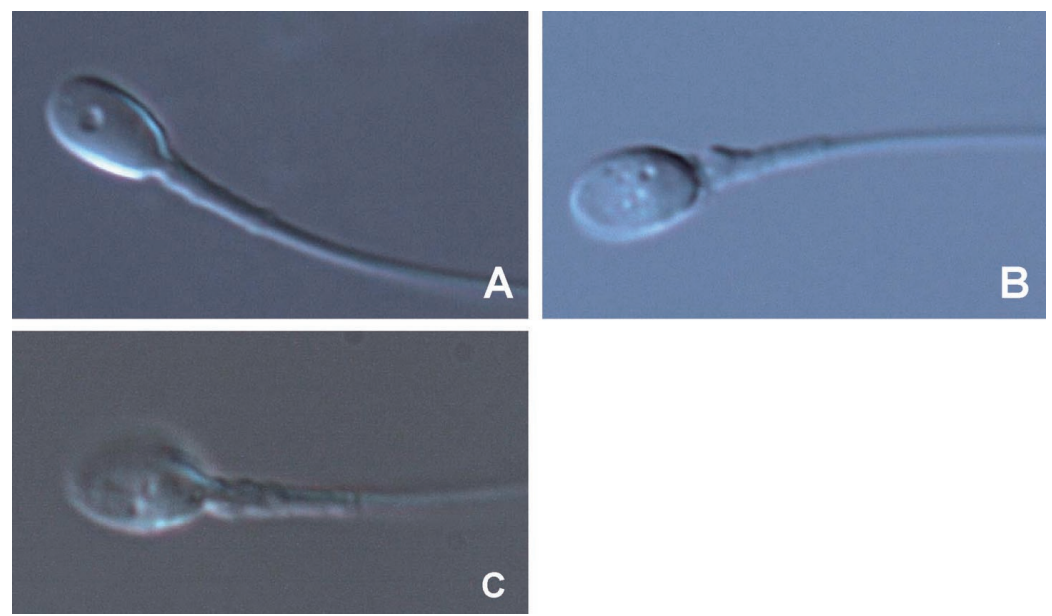

Fig. 7. The shape of sperm mid-piece as determined by IMSI.

A: Sperm with straight mid-pieces as selected by high magnification microscopy (Group 1) B: Sperm with tapering midpieces as selected by high magnification microscopy (Group 2) C: The sperm which do not fulfill the criteria of either A or B and are defined as "other".

[Original data, Terada, Y., Hasegawa, H., and Yaegashi, N.]

reported a case of infertility resulting from poor sperm centrosomal function due to Globozoospermia (Nakamura et al. 2002) and DFS (Rawe et al. 2002b). Both of these disorders have a morphological anomaly in the sperm mid-piece. These findings suggest that a morphologically abnormal sperm mid-piece is related to abnormal centrosomal function. As of now, however, no reports have examined the mid-piece in detail, likely because this region is difficult to visualize using normal magnification microscopy.

Microinjection with a motile sperm strictly selected by high-power $(6,000 \times)$ magnification microscopy, or intracytoplasmic morphologically selected sperm injection (IMSI), improves pregnancy rates in couples with repeated ICSI failure (Bartoov et al. 2002, 2003). IMSI also improves pregnancy outcomes over classic IVF-ICSI. Current reports examining IMSI focus on the selection of sperm based on head morphology (Berkovitz et al. 2005, 2006). We have focused on the shape of the sperm mid-piece, which contains the sperm centrosome. We examined sperm aster formation after injecting human sperm into bovine oocytes following selection by high magnification microscopy. High magnification microscopy permitted us to clearly identify small differences in mid-piece morphology (Fig. 7). We propose that it is possible to exclude these sperm with poor sperm centrosomal function with the aid of high magnification microscopy. The selection of sperm with straight midpieces may contribute to improved sperm aster formation. (Ugajin et al. 2010)

\section{Remarks}

We should like to acknowledge the contributions of the following individuals who contributed significantly to our understanding of sperm centrosomal function: Professor Kunihiro Okamura and Professor Takashi Murakami who are strong supporters of our research. We also express our thanks to Dr. Soichi Nakamura, Dr. Yumi Terunuma-Sato, Dr. Junko Kawano-Morita, Dr. Hiroshi Nabeshima, Dr. Masahito Tachibana, Dr. Yuki Shima-Morito, Dr. Tomoko Kakoi-Yoshimoto, Dr. Shinichi Hayasaka, Dr. Tomohisa Ugajin and Dr. Maiko Arai-Kikuchi. Finally, we would like to recognize Prof. Ryuzo Yanagimachi and his family (Prof. Toshitaka Horiuchi, Prof. Atsuo Ogura and other excellent scientists) for their continuous support and encouragement.

\section{Funding}

We gratefully acknowledge financial support from the Japan Society for the Promotion of Science to Y.T. and N.Y.

\section{References}

Asch, R., Simerly, C., Ord, T., Ord, V.A. \& Schatten, G. (1995) The stages at which human fertilization arrests: microtubule and chromosome configurations in inseminated oocytes which failed to complete fertilization and development in humans. Hum. Reprod., 10, 1897-1906.

Bartoov, B., Berkovitz, A., Eltes, F., Kogosowski, A., Menezo, Y. \& Barak, Y. (2002) Real-time fine morphology of motile human sperm cells is associated with IVF-ICSI outcome. $J$. Androl., 23, 1-8.

Bartoov, B., Berkovitz, A., Eltes, F., Kogosovsky, A., Yagoda, A., Lederman, H., Artzi, S., Gross, M. \& Barak, Y. (2003) Pregnancy rates are higher with intracytoplasmic morphologically selected sperm injection than with conventional intracytoplasmic injection. Fertil. Steril., 80, 1413-1419.

Berkovitz, A., Eltes, F., Ellenbogen, A., Peer, S., Feldberg, D. \& Bartoov, B. (2006) Does the presence of nuclear vacuoles in human sperm selected for ICSI affect pregnancy outcome? Hum. Reprod., 21, 1787-1790.

Berkovitz, A., Eltes, F., Yaari, S., Katz, N., Barr, I., Fishman, A. \& Bartoov, B. (2005) The morphological normalcy of the sperm nucleus and pregnancy rate of intracytoplasmic injection with morphologically selected sperm. Hum. Reprod., 20, 185-190.

Breed, W.G., Simerly, C., Navara, C.S., VandeBerg, J.L. \& Schatten, G. (1994) Microtubule configurations in oocytes, zygotes, and early embryos of a marsupial, Monodelphis domes- 
tica. Dev. Biol., 164, 230-240.

Brinkley, B.R. \& Goepfert, T.M. (1998) Supernumerary centrosomes and cancer: Boveri's hypothesis resurrected. Cell Motil. Cytoskeleton, 41, 281-288.

Cavilla, J.L., Kennedy, C.R., Byskov, A.G. \& Hartshorne, G.M. (2008) Human immature oocytes grow during culture for IVM. Hum. Reprod., 23, 37-45.

Chemes, H.E., Brugo, S., Zanchetti, F., Carrere, C. \& Lavieri, J.C. (1987) Dysplasia of the fibrous sheath: an ultrastructural defect of human spermatozoa associated with sperm immotility and primary sterility. Fertil. Steril., 48, 664-669.

Chemes, H.E. \& Rawe, V.Y. (2003) Sperm pathology: a step beyond descriptive morphology. Origin, characterization and fertility potential of abnormal sperm phenotypes in infertile men. Hum. Reprod. Update, 9, 405-428.

Dam, A.H., Feenstra, I., Westphal, J.R., Ramos, L., van, Golde, R.J. \& Kremer, J.A. (2007) Globozoospermia revisited. Hum. Reprod. Update, 13, 63-75.

Hayasaka, S., Terada, Y., Inoue, N., Okabe, M., Yaegashi, N. \& Okamura, K. (2007) Positive expression of the immunoglobulin superfamily protein IZUMO on human sperm of severely infertile male patients. Fertil. Steril., 88, 214-216.

Hewitson, L., Haavisto, A., Simerly, C., Jones, J. \& Schatten, G. (1997) Microtubule organization and chromatin configurations in hamster oocytes during fertilization and parthenogenetic activation, and after insemination with human sperm. Biol. Reprod., 57, 967-975.

Ikawa, M., Inoue, N. \& Okabe, M. (2008) Mechanisms of spermegg interactions emerging from gene-manipulated animals. Int. J. Dev. Biol., 52, 657-664.

Inoue, N., Ikawa, M., Isotani, A. \& Okabe, M. (2005) The immunoglobulin superfamily protein Izumo is required for sperm to fuse with eggs. Nature, 434, 234-238.

Iwao, Y., Yasumitsu, K., Narihira, M., Jiang, J. \& Nagahama, Y. (1997) Changes in microtubule structures during the first cell cycle of physiologically polyspermic newt eggs. Mol. Reprod. Dev., 47, 210-221.

Kim, N.H., Simerly, C., Funahashi, H., Schatten, G. \& Day, B.N. (1996) Microtubule organization in porcine oocytes during fertilization and parthenogenesis. Biol. Reprod., 54, 13971404.

Kovacic, B. \& Vlaisavljevic, V. (2000) Configuration of maternal and paternal chromatin and pertaining microtubules in human oocytes failing to fertilize after intracytoplasmic sperm injection. Mol. Reprod. Dev., 55, 197-204.

Levy, Y.Y., Lai, E.Y., Remillard, S.P., Heintzelman, M.B. \& Fulton, C. (1996) Centrin is a conserved protein that forms diverse associations with centrioles and MTOCs in Naegleria and other organisms. Cell Motil. Cytoskeleton, 33, 298-323.

Longo, F.J. (1976) Sperm aster in rabbit zygotes: its structure and function. J. Cell Biol., 69, 539-547.

Mailhes, J.B., Carabatsos, M.J., Young, D., London, S.N., Bell, M. \& Albertin, D.F. (1999) Taxol-induced meiotic maturation delay, spindle defects, and aneuploidy in mouse oocytes and zygotes. Mutat. Res., 423, 79-90.

Manandhar, G., Schatten, H. \& Sutovsky, P. (2005) Centrosome reduction during gametogenesis and its significance. Biol. Reprod., 72, 2-13.

Manipalviratn, S., DeCherney, A. \& Segars, J. (2009) Imprinting disorders and assisted reproductive technology. Fertil. Steril., 91, 305-315.

Mio, Y. \& Maeda, K. (2008) Time-lapse cinematography of dynamic changes occurring during in vitro development of human embryos. Am. J. Obstet. Gynecol., 199, 660 e1-e2.

Mitchison, T. \& Kirschner, M. (1984) Microtubule assembly nucleated by isolated centrosomes. Nature, 312, 232-237.

Morita, J., Terada, Y., Hosoi, Y., Fujinami, N., Sugimoto, M., Nakamura, S., Murakami, T., Yaegashi, N. \& Okamura, K. (2005) Microtubule organization during rabbit fertilization by intracytoplasmic sperm injection with and without sperm centrosome. Reprod. Med. Biol., 4, 169-177.

Morito, Y., Terada, Y., Nakamura, S., Morita, J., Yoshimoto, T., Murakami, T., Yaegashi, N. \& Okamura, K. (2005) Dynamics of microtubules and positioning of females pronucleus during bovine parthenogenesis. Biol. Reprod., 73, 935-941.

Murase, Y., Araki, Y., Mizuno, S., Kawaguchi, C., Naito, M., Yoshizawa, M. \& Araki, Y. (2004) Pregnancy following chemical activation of oocytes in a couple with repeated failure of fertilization using ICSI: case report. Hum. Reprod., 19 , 1604-1607.

Nakamura, S., Terada, Y., Horiuchi, T., Emuta, C., Murakami, T., Yaegashi, N. \& Okamura, K. (2001) Human sperm aster formation and pronuclear decondensation in bovine eggs following intracytoplasmic sperm injection using a Piezo-Driven Pipette: a novel assay for human sperm centrosomal function. Biol. Reprod., 65, 1359-1363.

Nakamura, S., Terada, Y., Horiuchi, T., Emuta, C., Murakami, T., Yaegashi, N. \& Okamura, K. (2002) Analysis of the human sperm centrosomal function and the oocyte activation ability in a case of globozoospermia, by ICSI into bovine oocytes. Hum. Reprod., 17, 2930-2934.

Nakamura, S., Terada, Y., Rawe, Y., Uehara, S., Morito, Y., Yoshimoto, T., Tachibana, M., Murakami, T., Yaegashi, N. \& Okamura, K. (2005) A trial to restore defective human sperm centrosomal function. Human. Reprod., 20, 1933-1937.

Nasr-Esfahani, M.H., Razavi, S., Javdan, Z. \& Tavalaee, M. (2008) Artificial oocyte activation in severe teratozoospermia undergoing intracytoplasmic sperm injection. Fertil. Steril., 90, 2231-2237.

Navara, C.S., First, N.L. \& Schatten, G. (1994) Microtubule organization in the cow during fertilization, polyspermy, parthenogenesis, and nuclear transfer: the role of the sperm aster. Dev. Biol., 162, 29-40.

Navara, C.S., First, N.L. \& Schatten, G. (1996) Phenotypic variations among paternal centrosomes expressed within the zygote as disparate microtubule lengths and sperm aster organization: correlations between centrosome activity and developmental success. Proc. Natl. Acad. Sci. USA, 93, 5384-5388.

Paffoni, A., Brevini, T.A., Somigliana, E., Restelli, L., Gandolfi, F. \& Ragni, G. (2007) In vitro development of human oocytes after parthenogenetic activation or intracytoplasmic sperm injection. Fertil. Steril., 87, 77-82.

Palermo, G.D., Colombero, L.T. \& Rosenwaks, Z. (1997) The human sperm centrosome is responsible for normal syngamy and early embryonic development. Rev. Reprod., 2, 19-27.

Palermo, G.D., Joris, H., Devroey, P. \& Van Steirteghem, A.C. (1992) Pregnancies after intracytoplasmic injection of single spermatozoon into an oocyte. Lancet, 340, 17-18.

Payne, C., Rawe, V., Ramalho-Santos, J., Simerly, C. \& Schatten, G. (2003) Preferentially localized dynein and perinuclear dynactin associate with nuclear pore complex proteins to mediate genomic union during mammalian fertilization. J. Cell Sci., 116, 4727-4738.

Picard, A., Karsenti, E., Dabauvalle, M.C. \& Doree, M. (1987) Release of mature starfish oocytes from interphase arrest by microinjection of human centrosomes. Nature, 327, 170-172.

Pinto-Correia, C., Collas, P., Ponce de Leon, F.A. \& Robl, J.M. (1993) Chromatin and microtubule organization in the first cell cycle in rabbit parthenotes and nuclear transplant embryos. Mol. Reprod. Dev., 34, 33-42.

Pinto-Correia, C., Poccia, D.L., Chang, T. \& Robl, J.M. (1994) Dephosphorylation of sperm midpiece antigens initiates aster formation in rabbit oocytes. Proc. Natl. Acad. Sci. USA, 91, 7894-7898.

Ramalho-Santos, J., Amaral, A., Brito, R., Freitas, M. \& AlmeidaSantos, T. (2004) Simultaneous analysis of cytoskeletal patterns and chromosome positioning in human fertilization failures. Fertil. Steril., 82, 1654-1659. 
Rawe, V.Y., Brugo, Olmedo, S., Nodar, F.N., Vitullo, A.D. (2002a) Microtubules and parental genome organisation during abnormal fertilisation in humans. Zygote, 10, 223-228.

Rawe, V.Y. \& Chemes, H. (2009) Exploring the cytoskeleton during intracytoplasmic sperm injection in humans. Methods Mol. Biol., 518, 189-206.

Rawe, V.Y., Olmedo, S.B., Nodar, F.N., Doncel, G.D., Acosta, A.A. \& Vitullo, A.D. (2000) Cytoskeletal organization defects and abortive activation in human oocytes after IVF and ICSI failure. Mol. Hum. Reprod., 6, 510-516.

Rawe, V.Y., Terada, Y., Nakamura, S., Chillik, C.F., Olmed, S.B. \& Chemes, H.E. (2002b) A pathology of the sperm centriole responsible for defective sperm aster formation, syngamy and cleavage. Hum. Reprod., 17, 2344-2349.

Rybouchkin, A., Dozortsev, D., Pelinck, M.J., De Sutter, P. \& Dhont, M. (1996) Analysis of the oocyte activating capacity and chromosomal complement of round-headed human spermatozoa by their injection into mouse oocyte. Hum. Reprod., 11, 2170-2175.

Rybouchkin, A., Van der Straeten, F., Quatacker, J., De Sutter, P. \& Dhont, M. (1997) Fertilization and pregnancy after assisted oocyte activation and intracytoplasmic sperm injection in a case of round-headed sperm associated with deficient oocyte activation capacity. Fertil. Steril., 68, 1144-1147.

Salisbury, J.L. (1995) Centrin, centrosomes, mitotic spindle poles. Curr. Opin. Cell Biol., 7, 39-45.

Sathananthan, A.H., Tatham, B., Dharmawardena, V., Grills, B., Lewis, I. \& Trounson, A. (1997) Inheritance of sperm centrioles and centrosomes in bovine embryos. Arch. Androl., 38, $37-48$.

Schatten, G. (1994) The centrosome and its mode of inheritance: the reduction of the centrosome during gametogenesis and its restoration during fertilization. Dev. Biol., 165, 299-335.

Schatten, G., Hewitson, L., Simerly, C., Sutovsky, P. \& Huszar, G. (1998) Cell and molecular challenges of ICSI: ART before science? J. Law Med. Ethic., 26, 29-37.

Schatten, G., Simerly, C. \& Schatten, H. (1985) Microtubule configurations during fertilization, mitosis, and early development in the mouse and the requirement for egg microtubule-mediated motility during mammalian fertilization. Proc. Natl. Acad. Sci. USA, 82, 4152-4156.

Schiebel, E. \& Bornens, M. (1995) In search of a function for centrins. Trends Cell Biol., 5, 197-201.

Seligman, J., Kosower, N.S., Weissenber, R. \& Shalgi, R. (1994) Thiol-disulfide status of human sperm proteins. J. Reprod. Fertil., 101, 435-443.

Shin, M.R. \& Kim, N.H. (2003) Maternal gamma $(\gamma)$-tubulin is involved in microtubule reorganization during bovine fertilization and parthenogenesis. Mol. Reprod. Dev., 64, 438-445.

Simerly, C., Wu, G.J., Zoran, S., Ord, T., Rawlins, R., Jones, J., Navara, C., Gerrity, M., Rinehart, J., Binor, Z. \& Schatten, G. (1995) The paternal inheritance of the centrosome, the cell's microtubule-organizing center, in humans, and the implications for infertility. Nat. Med., 1, 47-52.

Simerly, C., Zoran, S.S., Payne, C., Dominko, T., Sutovsky, P., Navara, C.S., Salisbury, L. \& Schatten, G. (1999) Biparental inheritance of $\gamma$-tubulin during human fertilization: molecular reconstitution of functional zygotic centrosomes in inseminated human oocytes and in cell-free extracts nucleated by human sperm. Mol. Biol. Cell, 10, 2955-2969.

Singh, G. (1992) Ultrastructural features of round-headed human spermatozoa. Int. J. Fertil., 37, 99-102.

Sutcliffe, A.G. \& Ludwig, M. (2007) Outcome of assisted reproduction. Lancet, 370, 351-359.

Suzuki, H., Saito, Y., Kagawa, N. \& Yang, X. (2003) In vitro fertilization of polyspermy in the pig: factors affecting fertilization rates and cytoskeletal reorganization of the oocyte. Microsc. Res. Tech., 61, 327-334.

Tateno, H. \& Kamiguchi, Y. (1999) Dithiothreitol induces sperm nuclear decondensation and protects against chromosome damage during male pronuclear formation in hybrid zygotes between Chinese hamster spermatozoa and Syrian hamster oocytes. Zygote, 7, 321-327.

Terada, Y. (2007) Functional analyses of the sperm centrosome in human reproduction: implications for assisted reproductive technique. Soc. Reprod. Fertil. Suppl., 63, 507-513.

Terada, Y., Hasegawa, H., Takahashi, A., Ugajin, T., Yaegashi, N. \& Okamura, K. (2009a) Successful pregnancy after oocyte activation by a calcium ionophore for a patient with recurrent intracytoplasmic sperm injection failure, with an assessment of oocyte activation and sperm centrosomal function using bovine eggs. Fertil. Steril., 91, 935 e11-14.

Terada, Y., Hasegawa, H., Ugajin, T., Murakami, T., Yaegashi, N. \& Okamura, K. (2009b) Microtubule organization during human parthenogenesis. Fertil. Steril., 91, 1271-1272.

Terada, Y., Hasegawa, H., Ugajin, T., Nabeshima, H., Suzuki, K., Yaegashi, N. \& Okamura, K. (2009c) Independent Spatial and Temporal Functions of Human Sperm Centrosomes After Dispermic Microinjection into Bovine Oocytes. J. Androl., 30, 559-565.

Terada, Y., Nakamura, S., Hewitson, L., Simerly, C., Horiuchi, T., Murakami, T., Okamura, K. \& Schatten, G. (2002) Human sperm aster formation after intracytoplasmic sperm injection with rabbit and bovine eggs. Fertil. Steril., 77, 1283-1284.

Terada, Y., Nakamura, S., Morita, J., Simerly, C., Hewitson, L., Murakami, T., Yaegashi, N., Schatten, G. \& Okamura, K. (2003) Intracytoplasmic sperm injection (ICSI): stiletto conception or a stab in the dark. Arch. Androl., 49, 169-177.

Terada, Y., Nakamura, S., Simerly, C., Hewitson, L., Murakami, T., Yaegashi, N., Okamura, K. \& Schatten, G. (2004a) Centrosomal function assessment in human sperm using heterologous ICSI with rabbit eggs: a new male factor infertility assay. Mol. Reprod. Dev., 67, 360-365.

Terada, Y., Nakamura, S., Morita, J., Tachibana, M., Morito, Y., Ito, K., Murakami, T., Yaegashi, N. \& Okamura, K. (2004b) Use of mammalian eggs for assessment of human sperm function: molecular and cellular analyses of fertilization by intracytoplasmic sperm injection. Am. J. Repro. Immunol., 51, 290-293.

Terada, Y., Simerly, C.R., Hewitson, L. \& Schatten, G. (2000) Sperm aster formation and pronuclear decondensation during rabbit fertilization and development of a functional assay for human sperm. Biol. Reprod., 62, 557-563.

Tomashov-Matar, R., Tchetchik, D., Eldar, A., Kaplan-Kraicer, R., Oron, Y. \& Shalgi, R. (2005) Strontium-induced rat egg activation. Reproduction, 130, 467-474.

Tsuiki, A., Hoshiai, H., Takahashi, K., Suzuki, M. \& Hoshi, K. (1986) Sperm-egg interactions observed by scanning electron microscopy. Arch Androl, 16, 35-47.

Ugajin, T., Terada, Y., Hasegawa, H., Nabeshima, H., Suzuki, K. \& Yaegashi, N. (2010) The shape of the sperm midpiece in intracytoplasmic morphologically selected sperm injection relates sperm centrosomal function. J. Assist. Reprod. Genet., [in press]

Van, Blerkom, J., Davis, P., Merriam, J. \& Sinclair, J. (1995) Nuclear and cytoplasmic dynamics of sperm penetration, pronuclear formation and microtubule organization during fertilization and early preimplantation development in the human. Hum. Reprod. Update, 1, 429-461.

Voribjev, I.A. \& Chenstov, Yu, S. (1982) Centrioles in the cell cycle. I. Epithelial cells. J. Cell Biol., 93, 938-949.

Wu, X. \& Palazzo, R.E. (1999) Differential regulation of maternal vs. paternal centrosomes. Proc. Natl. Acad. Sci. USA, 96, 1397-1402.

Yanagida, K., Katayose, H., Yazawa, H., Kimura, Y., Konnai, K. \& Sato, A. (1999) The usefulness of a piezo-micromanipulator in intracytoplasmic sperm injection in humans. Hum. Reprod., 14, 448-453.

Yan, W., Morozumi, K., Zhang, J., Ro, S., Park, C. \& Yanagimachi, 
R. (2008) Birth of mice after intracytoplasmic injection of single purified sperm nuclei and detection of messenger RNAs and MicroRNAs in the sperm nuclei. Biol. Reprod., 78, 896-902.

Yellera-Fernandez, M.D.M., Crozet, N. \& Ahmed-Ali, M. (1992)

Microtubule distribution during fertilization in the rabbit. Mol.
Reprod. Dev., 32, 271-276.

Yoshimoto-Kakoi, T., Terada, Y., Tachibana, M., Murakami, T., Yaegashi, N. \& Okamura, K. (2008) Assessing centrosomal function of infertile males using heterologous ICSI. Syst. Biol. Reprod. Med., 54, 135-142. 